\title{
Final Nail in the Coffin
}

\author{
Swati Jha ${ }^{1}$ \\ ${ }^{1}$ Sheffield Teaching Hospitals
}

July 4, 2021

\section{Title Page: Minicommentary}

This is a minicommentary on Offiah et al. "Long-term efficacy and complications of a multicentre randomised controlled trial comparing Retropubic and Transobturator Mid-Urethral Slings: a prospective observational study".

\section{Title: Final Nail in the Coffin.}

Author : Swati Jha (MD, FRCOG)

Consultant Gynaecologist and Honorary Senior Clinical Lecturer, Sheffield University. Subspecialist in Urogynaecology

Institute : Department of Urogynaecology, Sheffield Teaching Hospitals NHS Foundation Trust

Address : Sheffield Teaching Hospitals, Level 4, Jessop Wing, Tree Root Walk, Sheffield. S10 2SF

Swati.Jha1@nhs.net

$0044(0) 1142268568$

Fax : 01142268165

\section{Contribution:}

SJ: Conceived the article did the literature search and wrote the article

\section{Disclosures}

None relevant to this report

\section{Word Count: 499}

\section{Title: Final Nail in the Coffin}

Synthetic midurethral slings (MUS) for the management of stress urinary incontinence (SUI) have come under intense scrutiny of late. The NICE (National Institute of Health and Clinical Excellence) guidelines for the management of urinary incontinence over the past 15 years have continued to be cautious in their recommendations of the obturator MUS whilst being supportive of the retropubic (RP) MUS. In the 2006 NICE clinical guidance (CG 40), the advice was to make women aware of the "lack of long-term data" for obturator MUS. In 2013 (CG 171), when this guidance was updated, the advice remained unchanged.

Obturator MUS (Delorme. Prog Urol. 2001; 11 (6): 1306-1313) were developed to reduce the risk of bladder injury but have been associated with obturator neuropathies presenting as pain and restricted mobility of the thigh and hip adduction, along with sensory loss and pain across the anterior thigh. However, possibly the greatest issue with obturator MUS is the technical difficulty of complete removal, due to their placement deep in the obturator foramen. In the largest series of vaginal mesh removals for all indications, the commonest 
type of mesh removed was the obturator MUS (Miklos et al. Surg.Technol.Int. 2016; 29: 185-189) as well as having the highest complication rates.

This study by Offiah et al is a 12 year follow up study of the Monarc Trial (Freeman et al. Int Urogynecol J 2011; 23 (3); 279-86) which was a non-inferiority trial comparing the RP and obturator MUS and shows good follow up even a decade later. At 12 years, the overactive bladder (OAB) symptoms and recurrent UTI symptoms were similar in both groups, however it is difficult to know whether these were age related or a worsening of pre-existing OAB. Interestingly, this study showed a low incidence of vaginal and groin pain and equivalence in both types of MUS and this may be related to the fact that these procedures were performed by urogynaecologists with a good case load rather than the generalist. This highlights the importance of training which is a central theme in the Cumberlege report (First Do No Harm. https://www.immdsreview.org.uk/downloads/IMMDSReview_Web.pdf.

Accessed 12.06.21). This is also reflected in the PROSPECT trial (Glazener et al. Lancet 2017;389: 381-392).

However, the most significant finding is the stark difference in decline of success with time between obturator MUS compared to the RP. This gives us the long-term data we have been waiting for which shows beyond reasonable doubt that the obturator is inferior to the RP MUS with half the success at 12 years. This is probably the final nail in the coffin for obturator MUS and justifies the recent NICE recommendations from 2019, which states "do not offer a transobturator approach unless there are specific clinical circumstances in which the retropubic approach should be avoided."

What is urgently needed is similar long-term studies of the alternative SUI surgeries including colposuspension and autologous fascial slings so that patients can take all risks and benefits into account in reaching an informed decision about their preferred surgery. 